\title{
Ultrafast electron transport in layered semiconductors studied with femtosecond-laser photoemission
}

\author{
Armin Rettenberger and Paul Leiderer \\ Universität Konstanz, Fakultät für Physik, D-78434 Konstanz, Germany \\ Matthias Probst* and Richard Haight \\ IBM T. J. Watson Research Center, P.O. Box 218, Yorktown Heights, New York 10598
}

(Received 5 May 1997)

\begin{abstract}
Femtosecond-laser photoemission was used to investigate the electron dynamics in the layered semiconductors $\mathrm{MoSe}_{2}$ and $\mathrm{WSe}_{2}$. Photoexcitation with 200-fs pulses of $2.03 \mathrm{eV}$ light creates an electron gas with significant excess energy. Our measurements reveal a strong transient enhancement in the diffusive transport of the most energetic electrons relative to the conduction-band minimum. Additionally, we demonstrate that the surfaces of these layered chalcogenides are electronically passivated and we give an upper bound for the density of defect states within the band gap.
\end{abstract}

A detailed understanding of transient hot-electron effects on carrier transport in semiconductors is essential for various problems in modern physics and technology. It has been shown that carrier drift velocities and diffusivities can "overshoot" or exceed their equilibrium values whenever injected electrons are highly correlated in space or time. ${ }^{1,2}$ The fundamental time scale for many of these transient transport processes is in the picosecond time regime. Even in the absence of external electric fields, optical techniques can be used to create a hot nonequilibrium carrier distribution. While the mechanism of generating hot carriers by photoexcitation is quite different from heating by an electric field, the same relaxation and scattering processes apply in both cases. Thus the advent of ultrafast lasers has made it possible to probe these dynamic processes directly in the time domain on a femtosecond scale. Among time-resolved techniques, time-resolved photoemission spectroscopy is unique in that it directly measures the temporal evolution of photoexcited electrons. $^{3-6}$

In this paper we report on time-resolved photoemission investigations of the transient electron transport in the layered semiconductor chalcogenides $\mathrm{MoSe}_{2}$ and $\mathrm{WSe}_{2}$. These materials display interesting anisotropies in their abilities to transport electronic charge. ${ }^{7}$ At the same time, the strong absorption of visible radiation makes them attractive candidates for use in solar cells. ${ }^{8}$ In their crystalline form, these materials form trilayers, consisting of a metal layer bounded above and below by chalcogen atoms. Van der Waals forces bind the layers together to form the solid. The weak van der Waals interaction allows for easy cleavage of the crystal to reveal fresh material. Furthermore, as we will show, no states exist within the band gap of the semiconducting chalcogenides that we studied. The lack of surface states is consistent with our findings of a highly passivated surface formed upon cleavage of the material. As a result, we have been able to study the ultrafast bulk dynamics of electrons photoexcited into the conduction bands of layered semiconducting chalcogenides. We observe an extremely fast loss of photoexcited carriers in the first picoseconds, which can be as- cribed to an enhanced diffusion of carriers possessing large excess energy above the conduction-band minimum (cbm).

Our experimental approach to investigating the ultrafast bulk electron dynamics of $\mathrm{MoSe}_{2}$ and $\mathrm{WSe}_{2}$ involves the application of excite/probe femtosecond (fs) -laser photoemission spectroscopy. Although this technique has been described in detail elsewhere ${ }^{9}$ we give a short description here. An amplified dye-laser system operating at a repetition rate of $540 \mathrm{~Hz}$ produces $\sim 0.6-\mathrm{mJ}$ pulses of $200 \mathrm{fs}$ light at 610 nm. Roughly $20 \%$ of this light is used to photoexcite the sample under study while the remaining $80 \%$ is focussed into a burst of Ar gas at the output of a pulsed valve. The interaction of the intense $610 \mathrm{~nm}$ light with the high-density Ar generates odd multiple harmonics up to high orders. ${ }^{10-13}$ In the experiments to be discussed here, the 9th through the 15 th harmonics were used (18.3-30.5 eV). Separation of the harmonic orders was accomplished by angle tuning a 3-m grazing incidence grating that resides in a differentially pumped vacuum chamber coupled to a UHV analysis chamber. Overlap of the excitation pulse with the chosen harmonic produces photoelectron spectra of the excited sample. Electron detection and energy analysis is carried out with a parabolic-mirror-time-of-flight analyzer possessing a large solid angle of collection $(\sim 1.1 \mathrm{sr})$, which permits detection of the electrons throughout the crystal Brillouin zone.

For the investigations to be described, single crystal $n$-type $\mathrm{MoSe}_{2}$ and $p$-type $\mathrm{WSe}_{2}$ were grown with the vapor transport technique, ${ }^{8}$ crystallizing in the trigonal primatic $2 \mathrm{H}$ structure. Typical doping densities of the crystals are $10^{17} \mathrm{~cm}^{-3}$. Fresh surfaces were prepared by cleaving in ultrahigh vacuum (base pressure $=10^{-10}$ torr), which produced large optically defect-free areas. Low energy electron diffraction from these regions of the sample revealed sharp $1 \times 1$ patterns. All experiments were carried out on the defect-free regions of the samples we studied.

Absorption of a short, intense visible pulse of light by the sample results in the formation of an excited electron gas. The electron distribution thus created can evolve spatially 


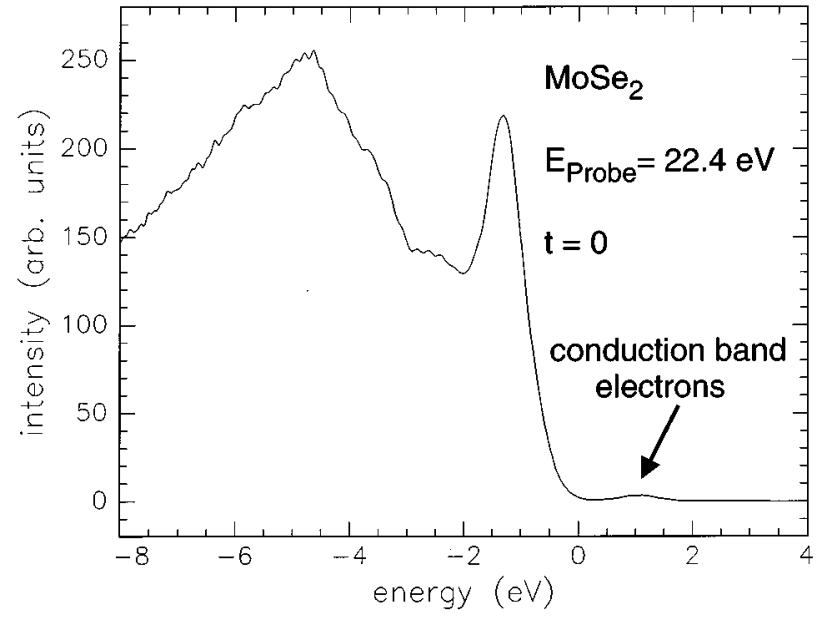

FIG. 1. Photoemission spectrum of photoexcited $\mathrm{MoSe}_{2}$ near $t$ $=0$.

through diffusion and ballistic transport away from the surface. In addition, electrons can transfer energy via electronphonon scattering, which results in energy and momentum relaxation of the electron gas. Figure 1 displays a representative photoelectron spectrum of photoexcited $\mathrm{MoSe}_{2}$, collected with 22.4-eV photons. The excite and probe pulses were coincident in time $(t=0)$. States below $0 \mathrm{eV}$ are normally occupied. The small feature $\sim 1 \mathrm{eV}$ above the valence edge is observed only when the system is photoexcited and results from the transient population of the conduction band. A careful analysis of the size of the band gap gives $1.1 \mathrm{eV}$, which compares well with results from optical and photoelectrochemical measurements. ${ }^{14}$ The broad and asymmetric peak indicates that a significant fraction of electrons possess excess energy above the cbm (see also Fig. 2). The calculated band structure of the hexagonal Brillouin zone (BZ) of $\mathrm{MoSe}_{2}$ indicates that it possesses an indirect gap of $1.1 \mathrm{eV}$ with the minimum direct gap of $1.4 \mathrm{eV}$ located at the $M$ point of the BZ. The cbm lies roughly halfway between the $\Gamma$ and $M$ points of the BZ. ${ }^{15,16}$ Photoexcitation produces a hotelectron gas distributed over a large fraction of the BZ, with a maximum excess energy relative to the cbm of $0.9 \mathrm{eV}$, giving rise to the broad conduction-band signal we observe. Similar results were obtained for $\mathrm{WSe}_{2}$ with minor differences due to the somewhat larger $1.2-\mathrm{eV}$ band gap. It is interesting to point out that the conduction-band signal was observed over a tuning range from 18.3 to $30.5 \mathrm{eV}$ in steps of $4 \mathrm{eV}$, although an enhancement in the intensity of the signal was observed from 22 to $26 \mathrm{eV}$, presumably due to final-state effects in the photoemission process.

Another interesting property of the layered materials can be observed by further inspection of Fig. 2. It is clear that the gap region is nearly devoid of emission. For our excite pulse fluence, the surface photoexcited electron density is estimated to be $2 \times 10^{12} / \mathrm{cm}^{2}$. Assuming that the defect states within the surface band gap emit with a similar probability as the conduction-band states we can give an upper limit of about $5 \times 10^{10} / \mathrm{cm}^{2}$ for the density of defect states. This shows directly that the van der Waals surfaces of $\mathrm{MoSe}_{2}$ and $\mathrm{WSe}_{2}$ are electronically extraordinarily well passivated.

In order to more fully investigate the dynamic behavior of

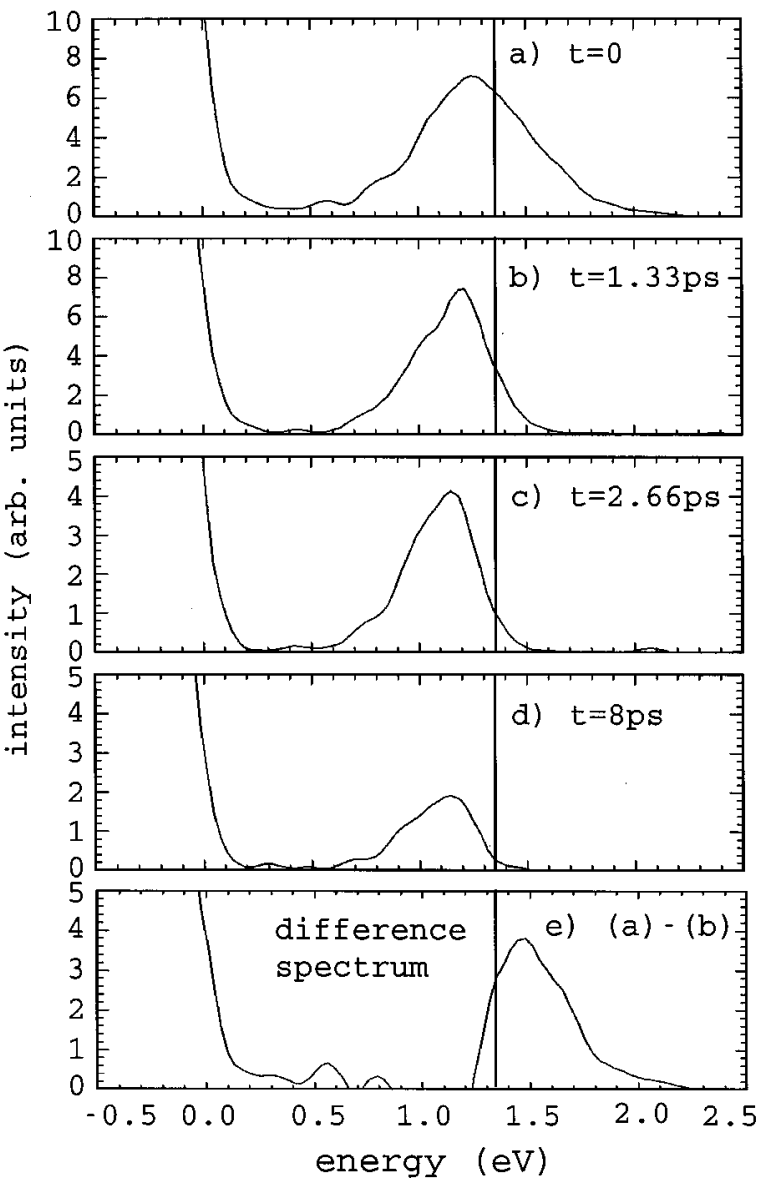

FIG. 2. (a)-(d) Spectra of the excited $\mathrm{WSe}_{2}$ population for different delays between excite and probe pulses. The vertical line is a guide to the eye for the loss of the highly excited electrons. (e) Differential change in the conduction-band peak between $t=0$ and $t=1.33$ ps.

the two systems under study, we monitored the total intensity of the conduction signal as a function of delay between the excite and probe pulses as shown in Fig. 3. In addition, a careful analysis of the evolution of the shape of the conduction signal was carried out as shown in Figs. 2. Figure 3 displays the normalized conduction-band (cb) emission intensity as a function of the delay time. For $\mathrm{MoSe}_{2}$ the signal intensity rises to a maximum within 400 fs. Remarkably, the signal decays by $66 \%$ in the following 2 ps with subsequent decay of the signal considerably slower. Qualitatively similar behavior is observed for $\mathrm{WSe}_{2}$ with a somewhat slower decay of the initial drop. A number of runs on different crystals and different spots on the same crystal yielded a high level of reproducibility in the observed time dependence. The overall behavior cannot be fit with a single exponential decay. Additionally, numerically solving the diffusion equation for a single diffusion coefficient yields an unsatisfactory fit to the data. Such analysis implies that more than one component contributes to the dynamics we observe.

While the time dependence of the overall intensity reveals interesting behavior, further insight can be gained from inspection of the shape of the conduction-band signal as a function of time. Figures 2(a)-2(d) display a panel consisting of four spectra showing the time evolution of the electron 


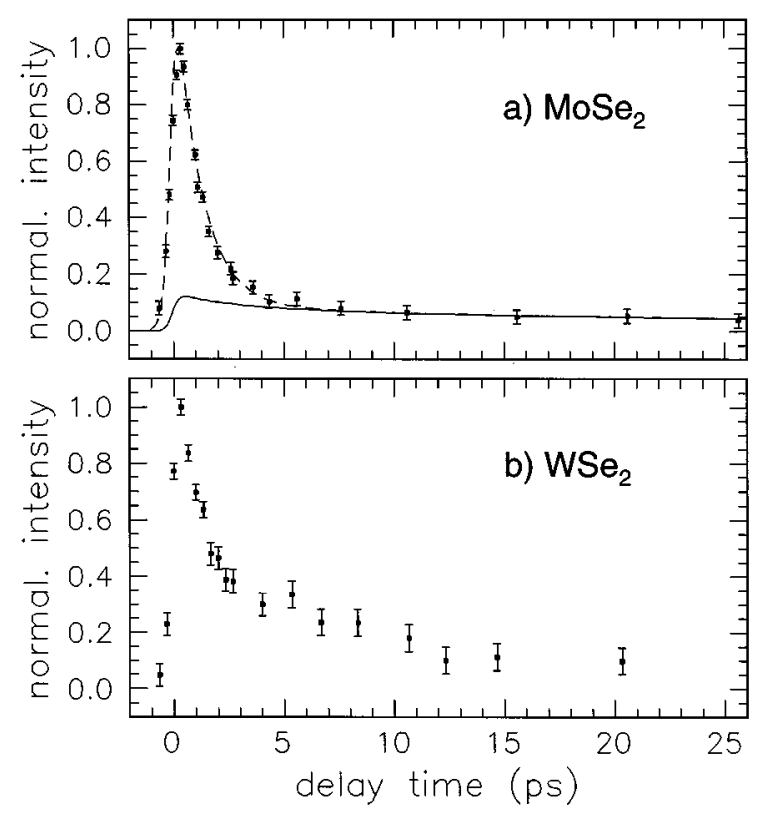

FIG. 3. Normalized emission intensity from the transiently occupied conduction band as a function of delay between excite and probe pulses for (a) $\mathrm{MoSe}_{2}$ and (b) $\mathrm{WSe}_{2}$. The solid curve is a fit based on the numerical solution of the diffusion equation for $D$ $=1 \mathrm{~cm}^{2} / \mathrm{s}$. The dashed curve is a fit to the ultrafast intensity loss with $\tau=1.1 \mathrm{ps}$.

population in $\mathrm{WSe}_{2}$. Figure 2(e) is the difference spectrum generated by subtracting Fig. 2(b) from 2(a). The top panel shows the signal collected at the temporal overlap of the excite and probe beams $(t=0)$. The peak is quite broad with a high-energy tail of electrons that approaches $2 \mathrm{eV}$ above the valence-band maximum. At $1.33 \mathrm{ps}$ the signal still has the same peak height but the high-energy edge has diminished significantly. At 2.66 ps a further retreat of the highenergy edge is observed concomitant with a reduction of the peak height. At 8 ps delay the signal has continued to decrease in intensity whereas the width has not changed significantly. Within the first 2-3 ps, the major change in the signal is due to the loss of the high-energy electrons. Several factors could contribute to the loss of these high-energy electrons. We can immediately rule out Auger recombination, which is too slow a process for the electron densities obtained in our experiment $\left(\leqslant 3 \times 10^{19} \mathrm{~cm}^{-3}\right)$ as well as radiative recombination, which is even slower for these indirect materials. ${ }^{17}$ In addition, the lack of observed surface or gap states implies that nonradiative electron-hole recombination is also absent. In particular, a possible explanation for the loss of high-energy electrons could be due to cooling of the distribution due to electron-phonon scattering. Such cooling would manifest itself as a narrowing of the distribution accompanied by a growth in height of the photoemission signal as the electrons degenerately fill all of the available states near the cbm. While such behavior has been observed in previous experiments ${ }^{18,19}$ this is not what is observed here. On the contrary, we observe a loss of the high-energy electrons with little change in intensity of the the lower-energy part of the spectrum. This change is reflected in the difference spectrum, Fig. 2(e), which shows that virtually all of the electron density loss occurs at energies beyond $1.3 \mathrm{eV}$ for a)

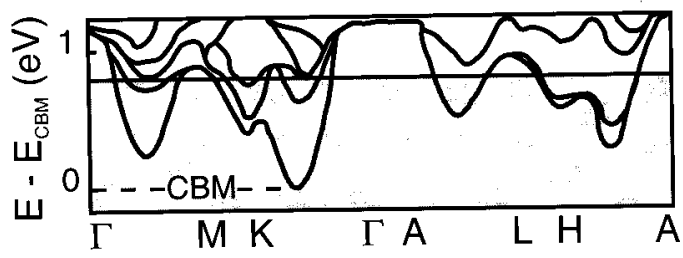

b)

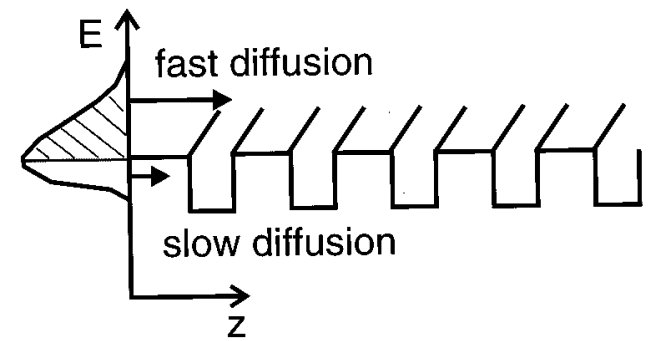

FIG. 4. (a) Structure of the conduction band of $\mathrm{WSe}_{2}$ (after Ref. 21). The hatched region indicates the energy range of the excited electrons. (b) Schematic representation of the energy-dependent transport of electrons from the surface.

times between $t=0$ and $t=1.33 \mathrm{ps}$. Although an overall reduction in intensity plus thermal equilibration could lead to similar observations we can rule out recombination on this time scale, as we discussed above. Since the escape depth of the photoemitted electrons we observe is $\sim 5-8 \AA$, the loss of the high-energy component of the cb signal is due to movement of the electrons away from the surface. We can conclude from these observations that the highest-energy electrons diffuse away from the surface much more rapidly than those near the cbm. If we correlate this observation with the decay in the overall intensity of the signal as shown in Fig. 3, we find that a significant contribution to the loss of the high-energy electrons may be found in the rapid transport away from the surface within the first 1-2 ps. Contributing to the loss of electrons near the surface, but at a slower rate, is the diffusion of the lower-energy electrons near the cbm. Diffusion of these electrons is observed in the slowly decaying tail in the intensity beyond 3-5 ps. Such an energy dependent diffusion process, while physically reasonable, has to our knowledge not been previously observed.

To get a more quantitative analysis, we fit a diffusion equation with a diffusion coefficient of $D=1 \mathrm{~cm}^{2} / \mathrm{s} .{ }^{20}$ This leads to the fit shown in Fig. 3 (solid line). The fit is unsatisfactory at early times but is good at later times when only lower-energy, more slowly diffusing electrons remain near the surface. In order to fit the fast early time component in Fig. 3(a), we have included an exponential term with a decay constant of $1.1 \mathrm{ps}$. While this term is purely phenomenological in nature, it emphasizes the existence of an ultrafast transport process that is operative in the presence of electrons possessing significant excess energy.

To understand this behavior we have to look somewhat closer into the physics of the diffusion process. Diffusion is a statistical process that depends upon the electrons scattering from phonons enough times that they undergo the diffusive random walk. The time it takes for the electrons to become "statistical" depends on the momentum relaxation time. For electrons with high excess energy there are many more final states to scatter into. The momentum relaxation time will be 
very fast but will slow down as the electrons lose energy. For $\mathrm{MoSe}_{2}$ and $\mathrm{WSe}_{2}$ this effect should be particularly pronounced since both materials are many-valley semiconductors. Figure 4(a) shows a sketch of the lower part of the conduction band of $\mathrm{WSe}_{2}$ (after Ref. 21). The hatched region indicates the maximum excess energy of the electrons in our experiment. There are many (sixfold) valleys available for the electrons to scatter into, and optical intervalley scattering will dominate the electron-lattice interaction. Additionally, scattering between nonequivalent valleys can lead to an intervalley contribution to the diffusivity. ${ }^{1,2}$ Electronic structure calculations reveal a conduction-band structure that is highly dispersive in the plane of the surface $(x-y)$ but that is significantly less dispersive in the perpendicular direction, consistent with the van der Waals nature of the intersheet bonding. Additionally, these calculations show that, close to the cbm, the character of the wave functions are mainly Mo- $d_{x y}, \mathrm{Se}-p_{x}$, and Se- $p_{y}$. At slightly higher energy Mo- $d_{z}^{2}$ dominates, resulting in greater overlap of the wave functions between the layers. ${ }^{16}$ This additionally supports the energy-dependent, higher mobility perpendicular to the layers. Therefore transport rates are expected to be highly energy dependent and can lead to the extremely fast loss in carrier density we observe. The effect of the energydependent wave-function properties and two-dimensional "confinement"' on the transport properties is despicted schematically in Fig. 4(b). Although the electronic structure of both materials is very similar, the somewhat greater enhancement for $\mathrm{MoSe}_{2}$ can be explained by the higher excess energy of the electrons due to the smaller band gap compared to $\mathrm{WSe}_{2}$.

To investigate transient carrier transport in high electric fields, Monte Carlo simulations ${ }^{1,2}$ have proven insightful, but only few experimental studies have been reported. ${ }^{22,23}$ Femtosecond-laser photoemission spectroscopy has enabled us to follow electron transport in the transient regime and to correlate directly, the enhanced diffusion with excess electron energy. It is often the case, particularly for indirect semiconductors, that achieving significant absorption of light (intense optical excitation) requires photons with energies much greater than the indirect gap, leading to electrons possessing considerable excess energies relative to the cbm. Thus in experiments such as, for example, short pulse laser annealing experiments, the early time diffusion of hot carriers can significantly extend the area of energy dissipation.

We wish to thank the group of Professor E. Bucher for the $\mathrm{MoSe}_{2}$ and $\mathrm{WSe}_{2}$ single crystals. We also thank J. Boneberg, M. Fischetti, K. Friemelt, and R. Möller for stimulating discussions.
*Present address: DLR, Institut für Technische Physik, Pfaffenwaldring 43, D-70569 Stuttgart, Germany.

${ }^{1}$ C. Jacobini and L. Reggiani, Rev. Mod. Phys. 55, 645 (1983).

${ }^{2}$ L. Reggiani, Hot-electron Transport in Semiconductors (Springer, New York, 1985).

${ }^{3}$ R. Haight et al., Phys. Rev. Lett. 54, 1302 (1985).

${ }^{4}$ J. Bokor et al., Phys. Rev. Lett. 57, 881 (1986).

${ }^{5}$ R. Haight, Surf. Sci. Rep. 21, 275 (1995).

${ }^{6}$ W. S. Fann et al., Phys. Rev. Lett. 68, 2834 (1992).

${ }^{7}$ R. C. Fivaz and Ph. E. Schmid, in Optical and Electrical Properties of Layered Semiconductors, edited by P. A. Lee (Reidel, Dordrecht, 1976).

${ }^{8}$ A. Aruchamy, Photoelectrochemistry and Photovoltaics of Layered Semiconductors (Kluwer Academic, Dordrecht, 1992).

${ }^{9}$ R. Haight and D. A. Peale, Rev. Sci. Instrum. 65, 1853 (1994).

${ }^{10}$ J. K. Crane et al., Opt. Lett. 17, 1256 (1992).

${ }^{11}$ A. L'Huillier and P. Balcou, Phys. Rev. Lett. 70, 774 (1993).

${ }^{12}$ J. L. Krause, K. J. Schafer, and K. C. Kulander, Phys. Rev. Lett. 68, 3535 (1992).
${ }^{13}$ J. J. Macklin, J. D. Kmetec, and C. L. Gordon III, Phys. Rev. Lett. 70, 766 (1993).

${ }^{14}$ K. K. Kam, C. L. Chang, and D. W. Lynch, J. Phys. C 17, 4031 (1984).

${ }^{15}$ R. Coehoorn et al., Phys. Rev. B 35, 6195 (1987).

${ }^{16}$ R. Coehoorn, C. Haas, and R. A. de Groot, Phys. Rev. B 35, 6203 (1987).

${ }^{17}$ W. Kautek, H. Gerischer, and H. Tributsch, J. Electrochem. Soc. 127, 2471 (1980).

${ }^{18}$ R. Haight and M. Baeumler, Phys. Rev. B 46, 1543 (1992).

${ }^{19}$ A. Rettenberger and R. Haight, Phys. Rev. Lett. 76, 1912 (1996).

${ }^{20}$ It is extremely difficult to measure the intrinsic electron mobility for layered chalcogenides perpendicular to the layers. Therefore we took the in-plane mobility of $100 \mathrm{~cm}^{2} \mathrm{~V} \mathrm{~s}$ and the anisotropy expected from band-structure calculations. This gives $D=1$ $\pm 0.5 \mathrm{~cm}^{2} / \mathrm{s}$ for room temperature.

${ }^{21}$ Th. Straub et al., Phys. Rev. B 53, R16 152 (1996).

${ }^{22}$ C. V. Shank et al., Appl. Phys. Lett. 38, 104 (1981).

${ }^{23}$ S. Laval, Microelectron. J. 13, 18 (1982). 